\title{
Application of Computer Simulation to Study the Features of the Austenite Isothermal Transformation in Steels
}

Yu.V. Yudin, M.V. Maisuradze, A.A. Kuklina, and P.D.Lebedev

Russia, Yekaterinburg, Mira Str., 19, Ural Federal University

\section{Abstract}

An algorithm was developed for the simulation of a phase transition in solid state which makes it possible to obtain the kinetic curves of transformation under different initial conditions (the number and arrangement of new phase nuclei, the distance between the nearest nuclei). The simulation results were analyzed using the KolmogorovJohnson-Mehl-Avrami equation and the corresponding coefficients were determined. The correlation between the simulation results and the experimental kinetics of the austenite isothermal transformation in alloyed steels was shown.

Keywords: computer simulation; modeling; phase transformation; kinetics; KJMA equation; logistic function; steel.
Yu.V. Yudin

yudinuv@rambler.ru

Received: 25 February 2019

Accepted: 9 April 2019

Published: 15 April 2019

Publishing services provided by

Knowledge E

(c) Yu.V. Yudin et al. This article is distributed under the terms of the Creative Commons

Attribution License, which permits unrestricted use and redistribution provided that the original author and source are credited.

Selection and Peer-review under the responsibility of the The Ural school-seminar of metal scientists-young researchers Conference Committee.

\section{Introduction}

During last decades the isothermal heat treatment of various steels is of a great importance in science and industry $[1,2]$ due to the development of new steel grades (TRIPaided steels [3], TBF steels [4], QP steels [5]) and to the implementation of new heat treatment technologies aimed to obtain bainite microstructure in steels (austempering [4], quenching-partitioning [5 - 7]). The main purpose of such investigations is to produce the steel parts possessing high strength and high ductility simultaneously. Therefore the detailed study of the processes taking place during the isothermal bainite transformation is of current interest along with the investigation of the factors affecting the bainite transformation kinetics.

The experimental kinetics of the isothermal austenite transformation (bainite and pearlite) usually consists of three characteristic stages [8, 9]: (1) initial acceleration of the transformation due to the formation of new nuclei and growth of previously formed nuclei; (2) deceleration of the transformation due to the site saturation and impingement of the growing volumes of new phase; (3) complete stop of the transformation due to 
the exhaustion of the untransformed volume or the absence of sites where new nuclei could be formed.

Mathematical description and analysis of the experimental kinetics of the austenite isothermal transformation may be performed using the following equations:

1. Kolmogorov-Johnson-Mehl-Avrami (KJMA) equation [10 - 14]

$$
P=1-\exp \left(-k \tau^{n}\right)
$$

where $\mathrm{P}$ - the fraction of the transformed austenite; $\mathrm{T}$ - time; $\mathrm{k}$ - the temperature dependent coefficient; $\mathrm{n}$ - the exponent.

2. Austin-Rickett equation [15]

$$
P=1-\left(k \tau^{n}+1\right)^{-1}
$$

3. Weibull function [16]

$$
P / P_{p}=1-\exp \left(-b(\ln (\tau))^{n}\right),
$$

where $\mathrm{P}_{p}$ - the maximum equilibrium transformation fraction obtained at the given temperature; $\mathrm{b}, \mathrm{n}$ - the equation parameters.

4. Logistic function [17]

$$
P / P_{p}=1-1 /[1+\exp (b+a \ln (\tau))],
$$

where $a, b$ - the constant coefficients for the given temperature of the isothermal transformation.

The choice of the equation for the mathematical description of the experimental data should provide the accuracy and adequacy of the calculation results. However the KJMA equation is often used even if the calculated transformation kinetics does not adequately fit the experimental data [ $18-20]$. This is related to the great fundamental basis of the coefficients of the KJMA equation [8, 9]. It is believed that $k$ is the parameter of the transformation rate determined by the transformation temperature and the activation energy and $\mathrm{n}$ is the exponent characterizing the parameters of the nucleation and growth of the new phase.

Lee and Kim [21] proposed the new generalized kinetic equation:

$$
d P / d \tau=k n \tau^{n-1}(1-P)^{c+1},
$$

where $\mathrm{c}$ is the impingement factor which accounts for the possible impingement modes of the growing volumes of the new phase. When $c=0$ the transformation slows down 
due to the change of the chemical composition of the parent phase and kinetics is well described by KJMA equation (soft impingement). When $c=1$ the hard impingement occurs due to the collision of the growing volumes of the new phase, and kinetics is described by Austin-Rickett equation.

The implementation of the different impingement modes may be attributed to the initial condition of the system (the concentration and the distribution of the nuclei of the new phase in the parent phase volume; the distance between the nuclei etc.). The present work is aimed at the analysis of the effect of the initial nuclei distribution on the transformation kinetics by means of the computer simulation. The correlation between the simulated transformation kinetics and the experimental kinetics of pearlite and bainite transformation in alloyed steels will be shown.

\section{Experimental}

The computer simulation of the phase transformation was performed using the "Matlab" software. The program was developed on the basis of the procedures for constructing the wave fronts in an isotropic medium [22]. The wave front was the interface between the transformed and untransformed parts of the single crystal. The modeling volume was a cube containing from 8 million to 64 million of the calculation nodes which can be in initial (untransformed) or new (transformed) condition. At the first stage of a simulation the program assigned the nodes of the new phase nuclei using random or presetted distribution. Then the procedure of the stepwise change of the phase condition of the nodes was performed. On every calculation step the transformation occurred in the nodes in initial condition which were directly bordering with the previously transformed volume. So the new phase grew layer by layer. The results of the simulation were listed in the text files which included: the distribution of the nuclei nodes before the start of the transformation; the distances between the nuclei; the number of the transformed nodes on every calculation step (transformation kinetics).

The analysis of the phase change kinetics was performed using KJMA equation (Eq. 1). The results were presented as the kinetic plots in logarithmic coordinates $\{\ln (-\ln (1-$ P)), $-\ln T\}$.

The following initial distributions of the new phase nuclei were chosen for the computer simulation: (1) in the vertices and the edges of the cube; (2) in the vertices and the faces of the cube; (3) randomly in the volume of the cube; (4) in the volume of the cube with the concentration gradient. 
The following simulation parameters were used: the volume of the cube was $8 \times 10^{6}$ nodes for the nuclei distributions 1, 2 and 3; $64 \times 10^{6}$ - for the nuclei distribution 4; the mean concentration of the new phase nuclei before the start of the transformation was $1,25 \times 10^{-3} \%$ for the nuclei distributions 1 and 2 ; from $1,25 \times 10^{-4} \%$ to $1,25 \times 10^{-2} \%$ for the nuclei distribution $3 ; 9,4 \times 10^{-4} \%$ for the nuclei distribution 4 . The projections of the new phase nuclei coordinates on the base plane of the cube are presented in Fig. 1 for the different nuclei distributions.
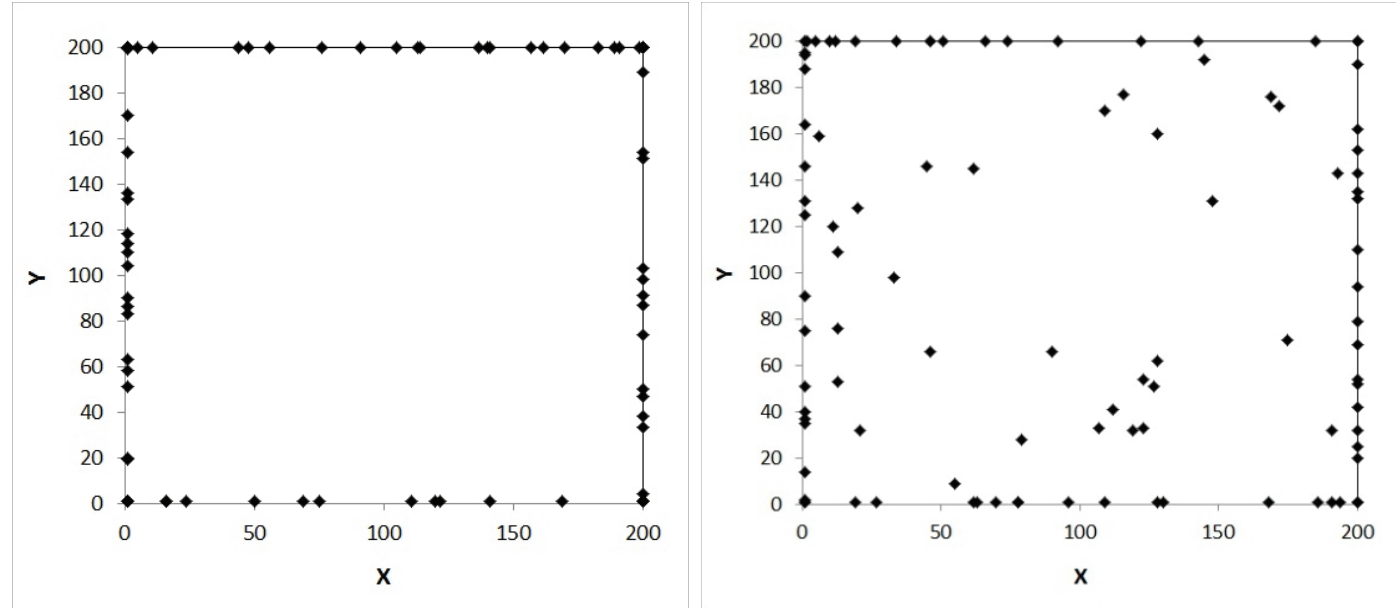

a

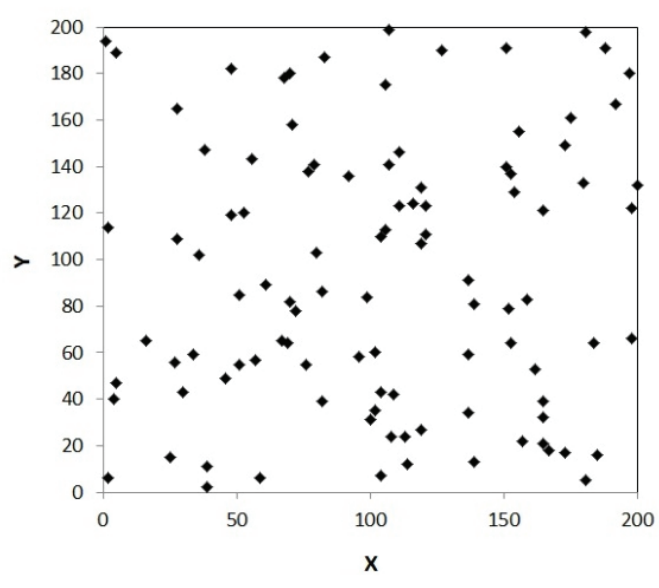

c

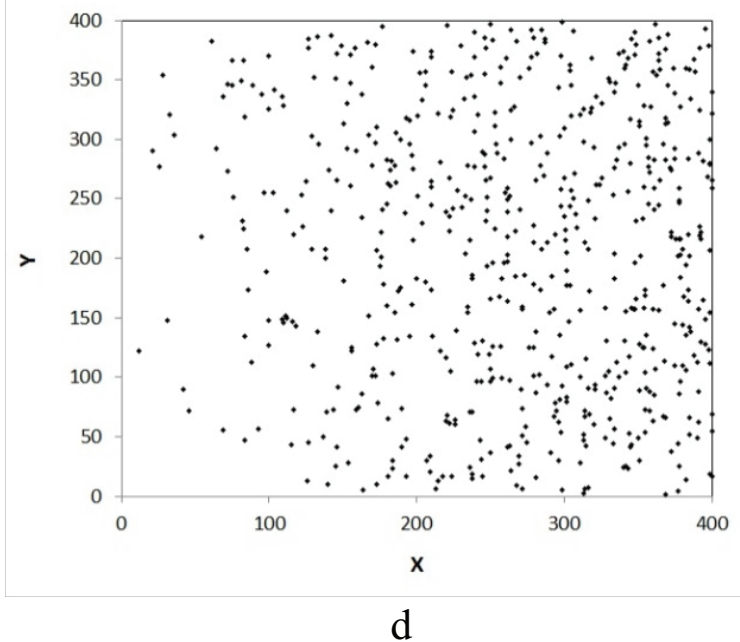

Figure 1: The projections of the coordinates of the new phase nuclei on the base plane of the cube before the transformation start: $a$ ) in the vertices and the edges of the cube; b) in the vertices and the faces of the cube; c) randomly in the volume of the cube (100 nuclei); $d$ ) in the volume of the cube with the concentration gradient.

The simulated kinetics of the phase transition was compared to the experimental austenite isothermal transformation kinetics in alloyed steels. The experimental data were obtained using the dilatometer Linseis L78 R.I.T.A. The cooling from the austenitization temperature $925{ }^{\circ} \mathrm{C}$ to the isothermal holding temperature $\left(670{ }^{\circ} \mathrm{C}\right.$ for the 
$38 \mathrm{Cr} 2 \mathrm{NiMoV}$ steel and $330{ }^{\circ} \mathrm{C}$ for the D6AC steel) was performed at the rate $30^{\circ} \mathrm{C} / \mathrm{s}$. The duration of the isothermal holding was at least $2 \mathrm{~h}$.

\section{Results and Discussion}

Fig. 2, a presents the typical kinetics of the simulated phase transformation in the cube $\left(8 \times 10^{6}\right.$ nodes) with various initial amount of new phase nuclei (10, 100 and 1000). The nuclei were randomly distributed through the volume of the cube (Fig. 1, c). It is obvious that the one hundredfold increase of the initial nuclei concentration led to the sufficient acceleration of the transformation and decrease of the total transformation time (approx. 5 times). The mathematical description of the simulated transformation kinetics using KJMA equation provided the good fit for almost entire transformation (Fig. 2, b). The deviation was observed only at the end of the transformation when the growing volume of the new phase approached the faces of the cube. The exponent of the KJMA equation $\mathrm{n} \approx 3$ and was the same for all the cases considered in Fig. 2 . The value of the $\ln (k)$ varied from $-13,54$ (for 10 nuclei) to $-9,04$ (for 1000 nuclei). Therefore the initial amount of the new phase nuclei did not significantly affect the value of the exponent $\mathrm{n}$. This agrees with the classical phase transformation theory stating that the value of the exponent $n$ is related with the shape of the growing particles of the new phase $[8,9]$.

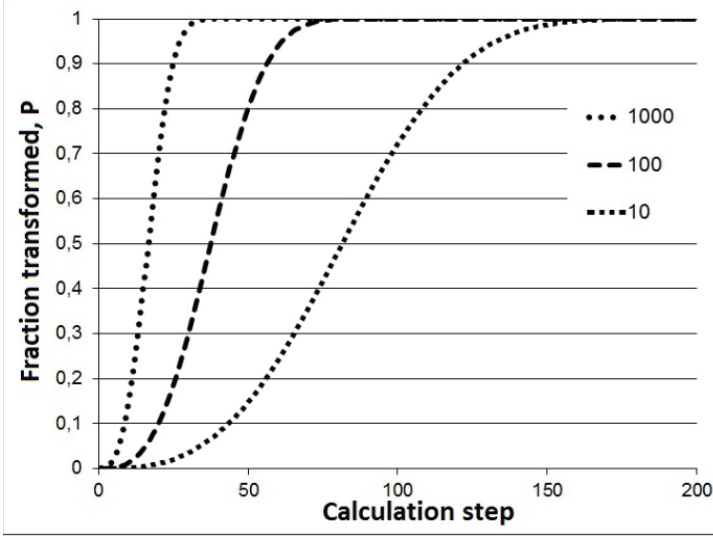

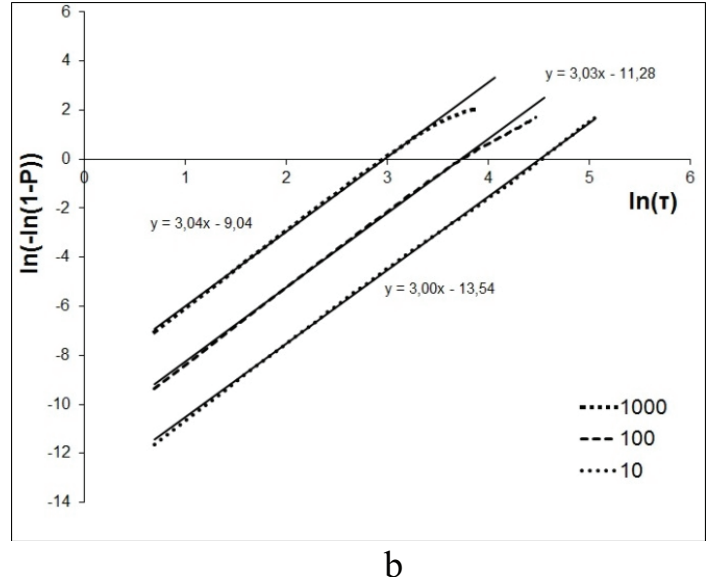

$\mathrm{b}$

Figure 2: The result of computer simulation of phase transformation with different initial concentration of the new phase nuclei (a) and the kinetics of phase transformation in logarithmic coordinates (b).

Somewhat different transformation kinetics was observed when the new phase nuclei were arranged in the vertices, edges and faces of the cube excluding the nuclei inside the cube (Fig. 1, a, b). When the nuclei were placed only in the vertices and the edges of the cube the exponent $n$ decreased to 2,3 (Fig. 3, a) and when the nuclei were arranged only in the vertices and the faces of the cube $-n=2,5$ (Fig. 3, b). The corresponding 
values of the $\ln (k)$ were in the range from $-10,6$ to $-10,3$. The obtained results show that the geometry of the nuclei distribution may significantly change the transformation kinetics. When the new phase nuclei were distributed non-uniformly throughout the volume of the cube (Fig. 1, d) the different transformation kinetics was observed (Fig. 3, c). From the simulation start to the $60-65 \%$ of the transformation the value of the exponent $n$ $=2,8$ and the value of the $\ln (k)=-11,0$. These values are close to the ones obtained for the random distribution of the nuclei throughout the volume of the cube. When the $60-$ $65 \%$ of the transformation was reached there was an inflection point on the logarithmic plot (Fig. 3, c) and the further transformation proceeded with the different values of the coefficients: $n=1,3$ and $\ln (k)=-5,2$.

The inflection on the kinetic plot (Fig. 3, c) was observed not only for the simulated transformation, but also for the real isothermal austenite transformations in steels. Fig. 4 presents the experimental isothermal transformation kinetics of the austenite in the 38Cr2NiMoV steel (austenite - pearlite transformation, Fig. 4, a) and D6AC steel (austenite - bainite transformation, Fig. 4, b). For the austenite - pearlite transformation in the $38 \mathrm{Cr} 2 \mathrm{NiMoV}$ steel the following values of the exponent $\mathrm{n}$ was obtained: from the transformation start until $73-78 \%$ of pearlite was formed $n=3,8$ and then $n=1,7$. For the austenite - bainite transformation in the D6AC steel $n=3,5$ until $83-85 \%$ of bainite was formed and then $\mathrm{n}$ decreased to 1,3 .

Some researchers $[23,24]$ associate the inflection on the kinetic plot with the change of the transformation "mode". However the similar inflections were observed both for the different experimental austenite transformations in steels (diffusion controlled austenite - pearlite transformation and combined diffusion controlled and diffusionless austenite - bainite transformation) and for the simulated transformation characterized by only one 'mode' of the phase transition (the volume of the new phase grows layer by layer per calculation step). Therefore the main factors determining the kinetics of the austenite transformation in steels are the distribution of the nuclei at the beginning of the transformation and, to some extent, the mean concentration of the nuclei.

The occurrence of the inflection on the kinetic plot may be attributed to the nonuniform distribution of the nuclei in the steel due to the local chemical or microstructure inhomogeneity. For example the $38 \mathrm{Cr} 2 \mathrm{NiMoV}$ steel had the microstructure inhomogeneity formed during the hot working of a sample and the D6AC steel possessed the banded microstructure after the hot rolling.

Thereby KJMA equation in its classical interpretation is not well suitable for the mathematical description of the isothermal austenite transformations observed in the commercial steels. The urgent matter nowadays is to find a method providing the most adequate 


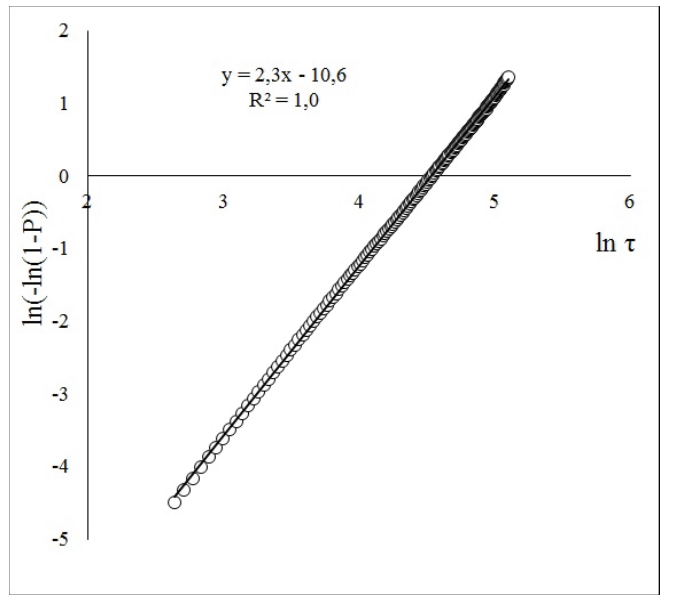

a

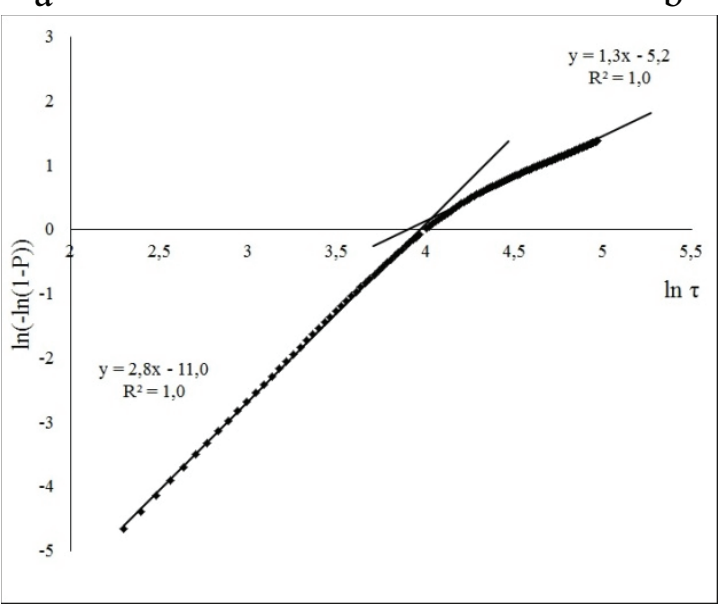

c

Figure 3: The simulated phase transformation kinetics for the initial arrangement of the nuclei in the vertices and the edges (a); in the vertices and the faces (b); for the non-uniform initial distribution.

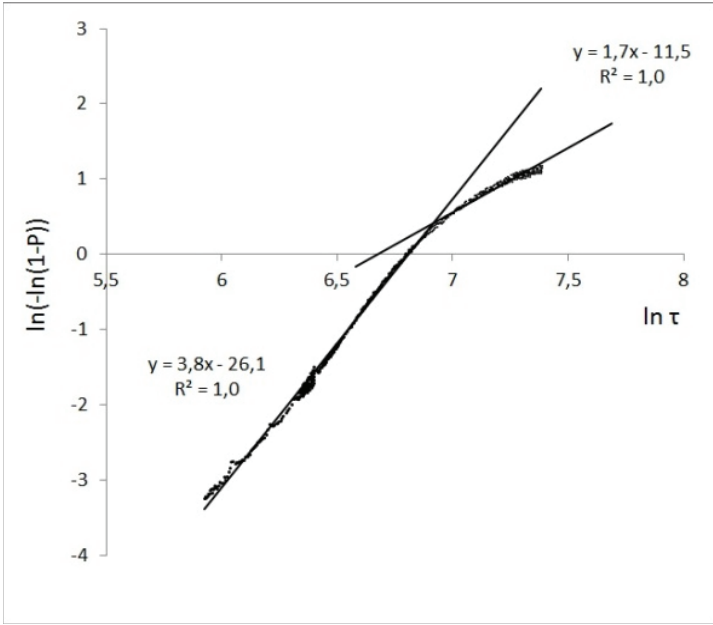

a

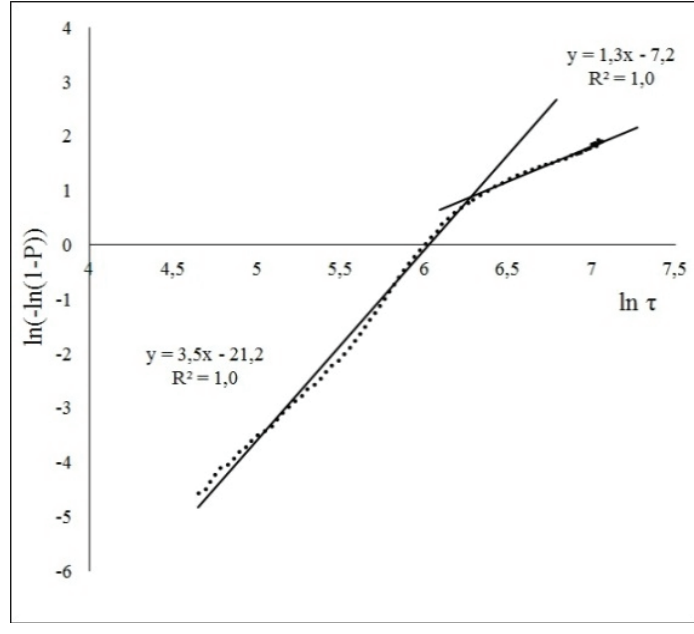

b

Figure 4: The experimental kinetics of isothermal austenite transformation in alloyed steels: a) pearlite transformation in $38 \mathrm{Cr} 2 \mathrm{NiMoV}$ steel, $670{ }^{\circ} \mathrm{C}$; b) bainite transformation in D6AC steel, $330^{\circ} \mathrm{C}$.

and convenient analysis of the experimental austenite transformation kinetics in steels. 
For example, when the logistic Eq. 4 [17] is used to analyze the same experimental and simulated transformation kinetics the resulting logarithmic plots do not have any inflection or other features. So the further analysis of the experimental data will be simplified and the wrong conclusions concerning the change of the transformation 'mode' will be avoided.

\section{Summary}

The algorithm for the computer simulation of the phase transition in the solid state is developed. The implemented procedures allow for estimation of the influence of the system's initial state on the transformation kinetics. The modeling volume is a cube containing up to $400 \times 400 \times 400$ calculation nodes.

The initial distribution of the new phase nuclei significantly affects the values of the coefficients $\mathrm{n}$ and $\mathrm{k}$ of the KJMA equation: when the nuclei are distributed uniformly throughout the cube $n=3$ regardless of the nuclei concentration; when the nuclei are arranged only in the vertices, the edges and the faces of the cube $n=2,3-2,5$; when there is the gradient of the nuclei concentration in the volume of the cube the exponent $\mathrm{n}$ varies during the transformation from 3 to 1,5 .

The kinetics of the simulated transformation (for the gradient distribution of the new phase nuclei) is similar to the kinetics of the isothermal pearlite and bainite transformation in commercial alloyed steels. This indicates that the change of the exponent $n$ during the austenite transformation may be attributed to the non-uniform distribution of the nuclei in the steel due to the chemical or microstructure inhomogeneity.

\section{Acknowledgements}

The work was supported by the Russian Federation Ministry of Education and Science (project № 11.1465.2014/K) and the Russian Federation Government Act № 211, contract №02.A03.21.0006.

\section{References}

[1] H. F. Lan, L. X. Du, Q. Li, Improvement of strength-toughness combination in austempered low carbon bainitic steel: The key role of refining prior austenitic grain size, J. Alloys Comp. 710 (2017) 702-710. 
[2] B. Avishan, M. Tavakolian, S. Yazdani, Two-step austempering of high performance steel with nanoscale microstructure, Mat. Sci. Eng. A. 693 (2017) 178-185.

[3] J. Kobayashi, D. Ina, N. Yoshikawa, K. Sugimoto, Effects of the Addition of Cr, Mo and $\mathrm{Ni}$ on the Microstructure and Retained Austenite Characteristics of $0.2 \% \mathrm{C}-$ $\mathrm{Si}-\mathrm{Mn}$ - Nb Ultrahigh-strength TRIP-aided Bainitic Ferrite Steels, ISIJ Int. 52 (2012) 1894-1901.

[4] L. Zhao, L. Qian, J. Meng, Below-Ms austempering to obtain refined bainitic structure and enhanced mechanical properties in low-C high-Si/Al steels, Scr. Mat. 112 (2016) 96-100.

[5] E. J. Seo, L. Cho, Y. Estrin, B. C. De Cooman, Microstructure-mechanical properties relationships for quenching and partitioning (Q\&P) processed steel, Acta Mat. 113 (2016) 124-139.

[6] A. J. Clarke, J. G. Speer, M. K. Miller, Carbon partitioning to austenite from martensite or bainite during the quench and partition (Q\&P) process: A critical assessment, Acta Mat. 56 (2008) 16-22.

[7] J. G. Speer, F. C. Rizzo, D. K. Matlock, D. V. Edmonds, The "Quenching and Partitioning" Process: Background and Recent Progress, Mat. Res. 8 (2005) 417-423.

[8] R. W. Cahn, P. Haasen, Physical Metallurgy, Vol. 2, North-Holland, 1996.

[9] J. W. Christian, The Theory of Transformations in Metal and Alloys, Pergamon, 2002.

[10] A.N. Kolmogorov, On the statistical theory of the crystallization of metals, Izv. AN SSSR. 3 (1937) 355-359 (in Russian).

[11] W. A. Johnson, R. F. Mehl, Reaction kinetics in process of nucleation and growth, Trans. AIME. 135 (1939) 416-468.

[12] M. Avrami, Kinetics of phase change I. General theory, J. Chem. Phys. 7 (1939) 11031112.

[13] M. Avrami, Kinetics of phase change II. Transformation-time relations for random distribution of nuclei, J. Chem. Phys. 8 (1940) 212-224.

[14] M. Avrami, Kinetics of phase change III. Granulation, phase change and microstructure, J. Chem. Phys. 9 (1941) 177-184.

[15] J. B. Austin, R. L. Rickett, Kinetics of the Decomposition of Austenite at Constant Temperature, Trans. AIMME. 964 (1939) 1-20.

[16] J. Cai, R. Liu, Weibull Mixture Model for Modeling Nonisothermal Kinetics of Thermally Stimulated Solid-State Reactions: Application to Simulated and Real Kinetic Conversion Data, J. Phys. Chem. B. 111 (2007) 10681-10686. 
[17] Yu. V. Yudin, M. V. Maisuradze, A. A. Kuklina, Describing the Isothermal Bainitic Transformation in Structural Steels by a Logistical Function, Steel Transl. 47 (2017) 213-218.

[18] N. X. Sun, X. D. Liu, K. Lu An explanation to the anomalous Avrami exponent, Scr. Mat. 34 (1996) 1201-1207.

[19] S. H. M. Azghandi, V. G. Ahmadabadi, Investigation on decomposition behavior of austenite under continuous cooling in vanadium microalloyed steel (30MSV6), Mat. Design. 88 (2015) 751-758.

[20] T. Jia, M. Militzer, Z. Y. Liu, General Method of Phase Transformation Modeling in Advanced High Strength Steels, ISIJ Int. 50 (2010) 583-590.

[21] E.-S. Lee, Y. G. Kim, A Transformation Kinetic Model And Its Application To Cu - Zn - Al Shape Memory Alloys. Isothermal Conditions, Acta Metal. Mat. 38 (1990) 16691676.

[22] P.D. Lebedev, A.A. Uspenskii, Program for constructing wave fronts and functions of the Euclidean distance to a compact nonconvex set, Certificate of state registration of the computer program №2017662074, 2017 (in Russian).

[23] M.A. Gervas'ev, O.V. Maslova, S.M. Illarionova, Kinetics of the bainite transformation in Cr-Ni-Mo steels with Al and Si, Izv. Vuz. Chern. Met. 57 (2014) 57-60 (in Russian).

[24] Z. Liu, R. O. Olivares, Y. Lei, Microstructural characterization and recrystallization kinetics modeling of annealing cold-rolled vanadium microalloyed HSLA steel, J. Alloys Comp. 679 (2016) 293-301. 